\title{
Planning with Stochastic Petri-Nets and Neural Nets
}

\author{
Nikolaos Bourbakis \\ Information Technology Research Institute, Wright State University, USA \\ bourbaki@cs.wright.edu
}

\begin{abstract}
This talk presents a synergistic methodology based on generalized stochastic Petri-nets (SPN) and neural nets for efficiently developing planning strategies. The SPN planning method generates global plans based on the states of the elements of the Universe of Discourse. Each plan includes all the possible conflict free planning paths for achieving the desirable goals under certain constraints occurred at the problem to be solved. The a neural network is used for searching the vectors of markings generated by the SPN reachability graph for the appropriate selection of plans. The SPN model presents high complexity issues, but at the same time offers to the synergic important features, such as stochastic modeling, synchronization, parallelism, concurrency and timing of events, valuable for developing plans under uncertainty. The neural network does contribute to the high complexity, but it offers learning capability to the synergy for future use. An example for coordinating two robotic arms under the constraints of time, space, and placement of the objects will be presented.
\end{abstract}

